Obsoletes RFCs: 776, 770, 762, 758,

$755,750,739,604,503,433,349$

Obsoletes IENs: 127, 117, 93

\author{
ASSIGNED NUMBERS
}

This Network Working Group Request for Comments documents the currently assigned values from several series of numbers used in network protocol implementations. This RFC will be updated periodically, and in any case current information can be obtained from Jon Postel. The assignment of numbers is also handled by Jon. If you are developing a protocol or application that will require the use of a link, socket, port, protocol, or network number please contact Jon to receive a number assignment.

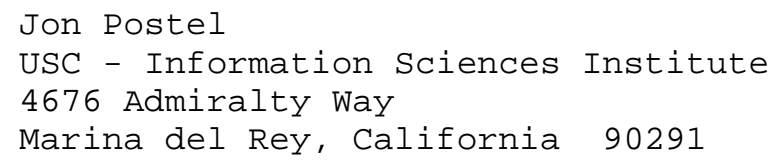

Most of the protocols mentioned here are documented in the RFC series of notes. The more prominent and more generally used are documented in the Protocol Handbook [17] prepared by the Network Information Center (NIC). Some of the items listed are undocumented. In all cases the name and mailbox of the responsible individual is indicated. In the lists that follow, a bracketed entry, e.g., [17,iii], at the right hand margin of the page indicates a reference for the listed protocol, where the number cites the document and the "iii" cites the person. 


\section{ASSIGNED NETWORK NUMBERS}

This list of network numbers is used in the internet address [33]. The Internet Protocol (IP) uses a 32 bit address and divides that address into a network part and a "rest" or local address part. The division takes 3 forms or classes.

The first type, or class a, of address has a 7-bit network number and a 24-bit local address. This allows 128 class a networks.

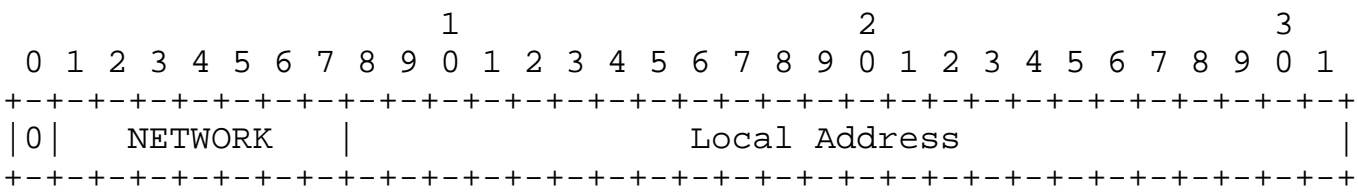

\section{Class A Address}

The second type, or class b, of address has a 14-bit network number and a 16-bit local address. This allows 16,384 class b networks.

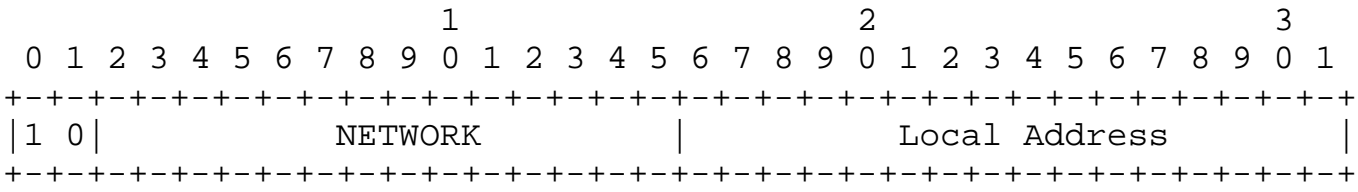

\section{Class B Address}

The third type, or class c, of address has a 21-bit network number and a 8-bit local address. This allows 2,097,152 class c networks.

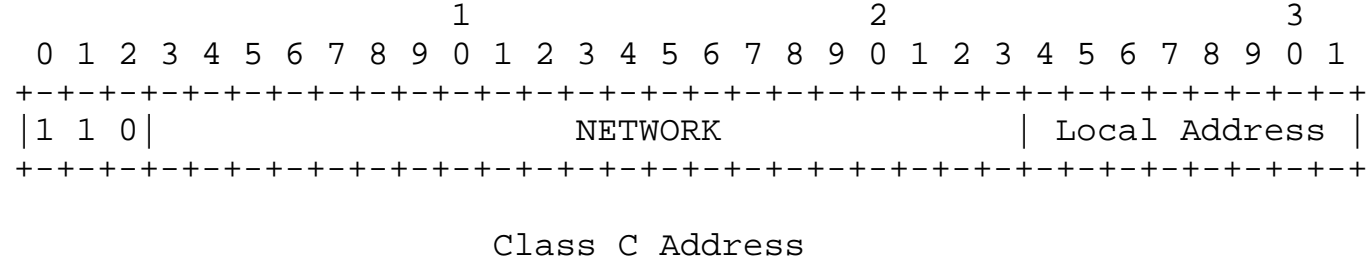

Class C Address

One notation for internet host addresses commonly used divides the 32-bit address into four 8-bit fields and specifies the value of each field as a decimal number with the fields separated by periods. For example, the internet address of ISIF is 010.020.000.052.

This notation will be used in the listing of assigned network 
numbers. The class a networks will have nnn.rrr.rrr.rrr, the class b networks will have nnn.nnn.rrr.rrr, and the class c networks will have nnn.nnn.nnn.rrr, where nnn represents part or all of a network number and rrr represents part or all of a local address or rest field.

Assigned Network Numbers

Class A Networks

\begin{tabular}{|c|c|c|}
\hline Internet Address & Name & References \\
\hline------ & -- & ------- \\
\hline $000 . \operatorname{rrg} \operatorname{srr} \operatorname{srr}$ & & Reserved \\
\hline 001 .rrr.rrr.rrr & $\mathrm{BBN}-\mathrm{PR}$ & BBN Packet Radio Network \\
\hline 002 .rrr.rrr.rrr & $S F-P R-1$ & SF Packet Radio Network (1) \\
\hline $003 . \operatorname{rrg} \operatorname{srr} \operatorname{srr}$ & $\mathrm{BBN}-\mathrm{RCC}$ & BBN RCC Network \\
\hline $004 . r r r . r r r . r r r$ & SATNET & Atlantic Satellite Network \\
\hline $005 . \operatorname{rrr}$.rre.rrr & SILL-PR & Ft. Sill Packet Radio Network [JEM] \\
\hline $006 . \operatorname{rrr} \operatorname{srr} \cdot \operatorname{rrr}$ & $S F-P R-2$ & SF Packet Radio Network (2) \\
\hline $007 . r r r . r r r . r r r$ & CHAOS & MIT CHAOS Network \\
\hline $008 \cdot \operatorname{rrr} \operatorname{srr} \cdot \operatorname{rrr}$ & CLARKNET & SATNET subnet for Clarksburg[DM11] \\
\hline $009 . \operatorname{rr}$.rrr.rrr & BRAGG-PR & Ft. Bragg Packet Radio Net \\
\hline $010 . \operatorname{rrr} \cdot \operatorname{rr} \operatorname{srr}$ & ARPANET & {$[17,1, \mathrm{VGC}]$} \\
\hline $011 . r r r . r r r . r r r$ & UCLNET & University College London \\
\hline 012 .rrr.rrr.rrr & CYCLADES & CYCLADES \\
\hline 013.rrr.rrr.rrr & & Unassigned \\
\hline 014 .rrr.rrr.rrr & TELENET & TELENET \\
\hline 015.rrr.rrr.rrr & EPSS & British Post Office EPSS \\
\hline $016 . \operatorname{rrr} \cdot \operatorname{rrr} \operatorname{srr}$ & DATAPAC & DATAPAC \\
\hline 017.rrr.rrr.rrr & TRANSPAC & TRANSPAC \\
\hline $018 \cdot \operatorname{rrr} \cdot \operatorname{rrr} \cdot \operatorname{rrr}$ & LCSNET & MIT LCS Network \\
\hline 019.rrr.rrr.rrr & TYMNET & TYMNET \\
\hline $020 . \operatorname{rrr} \cdot \operatorname{rrr} \operatorname{rrr}$ & $\mathrm{DC}-\mathrm{PR}$ & D.C. Packet Radio Network \\
\hline $021 \cdot \operatorname{rrr} \cdot \operatorname{rrr} \operatorname{rrr}$ & EDN & DCEC EDN \\
\hline 022 .rrr.rrr.rrr & DIALNET & {$[26,16, \mathrm{MRC}]$} \\
\hline 023.rrr.rrr.rrr & MITRE & MITRE Cablenet \\
\hline 024 .rre.rrr.rrr & BBN-LOCAL & BBN Local Network \\
\hline $025 \cdot \operatorname{rrr} \cdot \operatorname{rrr} \operatorname{rrr}$ & RSRE-PPSN & RSRE / PPSN \\
\hline 026.rrr.rrr.rrr & AUTODIN-II & AUTODIN II \\
\hline 027 .rrr.rrr.rrr & NOSC-LCCN & NOSC / LCCN \\
\hline $028 \cdot \operatorname{rrr} \cdot \operatorname{rr} \operatorname{srr}$ & WIDEBAND & Wide Band Satellite Network [CJW2] \\
\hline 029.rrr.rrr.rrr & DCN-COMSAT & COMSAT Dist. Comp. Network \\
\hline 030.rrr.rre.rrr & $\mathrm{DCN}-\mathrm{UCL}$ & UCL Dist. Comp. Network \\
\hline $031 . \operatorname{rrr} \operatorname{srr} \operatorname{rrr}$ & $\mathrm{BBN}-\mathrm{SAT}-\mathrm{TEST}$ & BBN SATNET Test Network \\
\hline 032 .rre.rre.rrr & $\mathrm{UCL}-\mathrm{CR} 1$ & UCL Cambridge Ring 1 \\
\hline 033.rrr.rrr.rrr & $\mathrm{UCL}-\mathrm{CR} 2$ & UCL Cambridge Ring 2 \\
\hline 034 .rrr.rrr.rrr & MATNET & Mobile Access Terminal Net \\
\hline $035 . \operatorname{rrr} \operatorname{srr} \operatorname{rrr}$ & NULL & UCL/RSRE Null Network \\
\hline
\end{tabular}




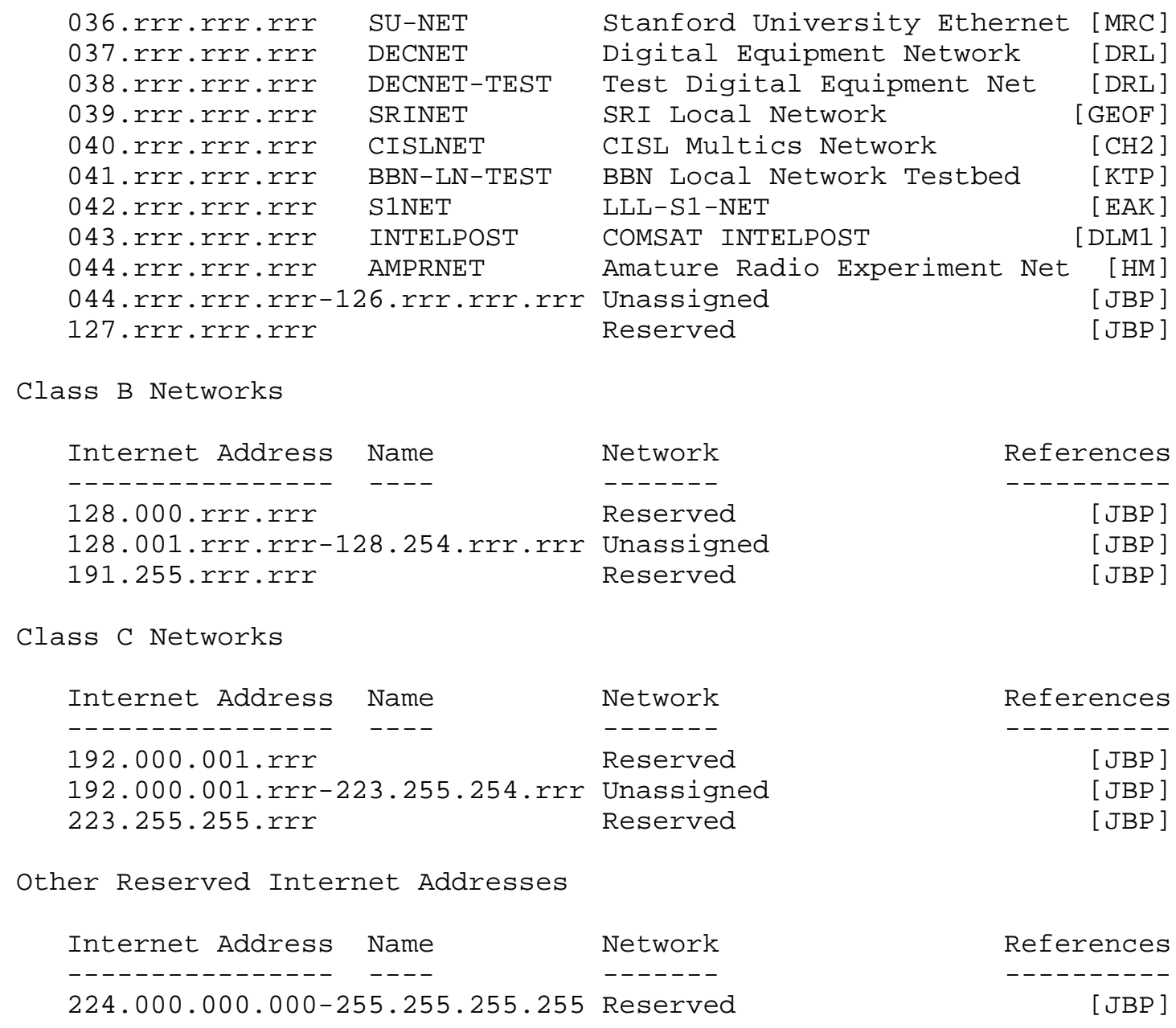


Internet Version Numbers

\section{ASSIGNED INTERNET VERSION NUMBERS}

In the Internet Protocol (IP) [33] there is a field to identify the version of the internetwork general protocol. This field is 4 bits in size.

Assigned Internet Version Numbers

$\begin{array}{rcl}\text { Decimal } & \text { Octal } & \text { Version } \\ ------ & ----- & ------ \\ 0 & 0 & \text { Reserved } \\ 1-3 & 1-3 & \text { Unassigned } \\ 4 & 4 & \text { Internet Protocol } \\ 5 & 5 & \text { ST Datagram Mode } \\ 6-14 & 6-16 & \text { Unassigned } \\ 15 & 17 & \text { Reserved }\end{array}$

References
$[\mathrm{JBP}]$
$[\mathrm{JBP}]$
$[33, \mathrm{JBP}]$
$[20, \mathrm{JWF}]$
$[\mathrm{JBP}]$
$[\mathrm{JBP}]$




\section{ASSIGNED INTERNET PROTOCOL NUMBERS}

In the Internet Protocol (IP) [33] there is a field, called Protocol, to identify the the next level protocol. This is an 8 bit field.

Assigned Internet Protocol Numbers

\begin{tabular}{|c|c|c|c|}
\hline Decimal & Octal & Protocol Numbers & References \\
\hline------- & ----- & ---------------- & ---------1 \\
\hline 0 & 0 & Reserved & {$[\mathrm{JBP}]$} \\
\hline 1 & 1 & ICMP & {$[53, \mathrm{JBP}]$} \\
\hline 2 & 2 & Unassigned & {$[\mathrm{JBP}]$} \\
\hline 3 & 3 & Gateway-to-Gateway & {$[48,49, \mathrm{VMS}]$} \\
\hline 4 & 4 & CMCC Gateway Monitoring Message & {$[18,19, \mathrm{DFP}]$} \\
\hline 5 & 5 & $\mathrm{ST}$ & {$[20, \mathrm{JWF}]$} \\
\hline 6 & 6 & $\mathrm{TCP}$ & {$[34, \mathrm{JBP}]$} \\
\hline 7 & 7 & UCL & {$[\mathrm{PK}]$} \\
\hline 8 & 10 & Unassigned & [ JBP ] \\
\hline 9 & 11 & Secure & [VGC] \\
\hline 10 & 12 & BBN RCC Monitoring & [VMS ] \\
\hline 11 & 13 & NVP & {$[12, \mathrm{DC}]$} \\
\hline 12 & 14 & PUP & {$[4, \mathrm{EAT} 3]$} \\
\hline 13 & 15 & Pluribus & [RDB2 ] \\
\hline 14 & 16 & Telenet & [RDB2 ] \\
\hline 15 & 17 & XNET & {$[25, \mathrm{JFH} 2]$} \\
\hline 16 & 20 & Chaos & {$[\mathrm{MOON}]$} \\
\hline 17 & 21 & User Datagram & {$[42, \mathrm{JBP}]$} \\
\hline 18 & 22 & Multiplexing & {$[13, \mathrm{JBP}]$} \\
\hline 19 & 23 & $\mathrm{DCN}$ & [DLM1] \\
\hline 20 & 24 & TAC Monitoring & {$[55, \mathrm{RH} 6]$} \\
\hline $21-62$ & $25-76$ & Unassigned & {$[\mathrm{JBP}]$} \\
\hline 63 & 77 & any local network & {$[\mathrm{JBP}]$} \\
\hline 64 & 100 & SATNET and Backroom EXPAK & [DM11] \\
\hline 65 & 101 & MIT Subnet Support & {$[\mathrm{NC} 3]$} \\
\hline $66-68$ & $102-104$ & Unassigned & {$[\mathrm{JBP}$} \\
\hline 69 & 105 & SATNET Monitoring & {$[\mathrm{DM} 11$} \\
\hline 70 & 106 & Unassigned & {$[\mathrm{JBP}$} \\
\hline 71 & 107 & Internet Packet Core Utility & {$[\mathrm{DM} 11$} \\
\hline $72-75$ & $110-113$ & Unassigned & {$[\mathrm{JBP}$} \\
\hline 76 & 114 & Backroom SATNET Monitoring & {$[\mathrm{DM} 1]$} \\
\hline 77 & 115 & Unassigned & {$[\mathrm{JBP}$} \\
\hline 78 & 116 & WIDEBAND Monitoring & {$[\mathrm{DM} 11$} \\
\hline 79 & 117 & WIDEBAND EXPAK & {$[\mathrm{DM} 1]$} \\
\hline $80-254$ & $120-376$ & Unassigned & {$[\mathrm{JBE}$} \\
\hline 255 & 377 & Reserved & {$[\mathrm{JBE}$} \\
\hline
\end{tabular}




\begin{abstract}
ASSIGNED PORT or SOCKET NUMBERS
Ports are used in the TCP [34] and sockets are used in the AHHP $[28,17]$ to name the ends of logical connections which carry long term conversations. For the purpose of providing services to unknown callers a service contact socket is defined. This list specifies the port or socket used by the server process as its contact socket. In the AHHP an Initial Connection Procedure ICP $[39,17]$ is used between the user process and the server process to make the initial contact and establish the long term connections leaving the contact socket free to handle other callers. In the TCP no ICP is necessary since a port may engage in many simultaneous connections.

To the extent possible these same port assignments are used with UDP [42].

The assigned ports/sockets use a small part of the possible port/socket numbers. The assigned ports/sockets have all except the low order eight bits cleared to zero. The low order eight bits are specified here.
\end{abstract}

Socket Assignments:

General Assignments:

$\begin{array}{lll}\text { Decimal } & \text { Octal } & \text { Description } \\ ------ & ----- & --------- \\ 0-63 & 0-77 & \text { Network Wide Standard Function } \\ 64-131 & 100-203 & \text { Hosts Specific Functions } \\ 132-223 & 204-337 & \text { Reserved for Future Use } \\ 224-255 & 340-377 & \text { Any Experimental Function }\end{array}$


Port or Socket Numbers

Specific Assignments:

Network Standard Functions

\begin{tabular}{|c|c|c|c|}
\hline Decimal & Octal & Description & References \\
\hline------- & ----- & ----------- & ---------- \\
\hline 1 & 1 & Old Telnet & {$[40, \mathrm{JBP}]$} \\
\hline 3 & 3 & Old File Transfer & $, 11,24, \mathrm{JBP}]$ \\
\hline 5 & 5 & Remote Job Entry & {$[6,17, \mathrm{JBP}]$} \\
\hline 7 & 7 & Echo & {$[35, \mathrm{JBP}]$} \\
\hline 9 & 11 & Discard & {$[32, \mathrm{JBP}]$} \\
\hline 11 & 13 & Who is on or SYSTAT & [ JBP ] \\
\hline 13 & 15 & Date and Time & {$[\mathrm{JBP}]$} \\
\hline 15 & 17 & Who is up or NETSTAT & {$[\mathrm{JBP}]$} \\
\hline 17 & 21 & Short Text Message & {$[\mathrm{JBP}]$} \\
\hline 19 & 23 & Character generator or TTYTST & {$[31, \mathrm{JBP}]$} \\
\hline 21 & 25 & New File Transfer & {$[36, \mathrm{JBP}]$} \\
\hline 23 & 27 & New Telnet & {$[41, \mathrm{JBP}]$} \\
\hline 25 & 31 & SMTP & {$[54, \mathrm{JBP}]$} \\
\hline 27 & 33 & NSW User System w/COMPASS FE & {$[14, \mathrm{RHT}]$} \\
\hline 29 & 35 & MSG-3 ICP & {$[29, \mathrm{RHT}]$} \\
\hline 31 & 37 & MSG-3 Authentication & {$[29, \mathrm{RHT}]$} \\
\hline 33 & 41 & Unassigned & {$[\mathrm{JBP}]$} \\
\hline 35 & 43 & IO station Spooler & {$[\mathrm{JBP}]$} \\
\hline 37 & 45 & Time Server & {$[22, \mathrm{JBP}]$} \\
\hline 39 & 47 & Unassigned & {$[\mathrm{JBP}]$} \\
\hline 41 & 51 & Graphics & {$[46,17, \mathrm{JBP}]$} \\
\hline 42 & 52 & Name Server & {$[38, \mathrm{JBP}]$} \\
\hline 43 & 53 & WhoIs & {$[\mathrm{JAKE}]$} \\
\hline 45 & 55 & Message Processing Module & {$[37, \mathrm{JBP}]$} \\
\hline 47 & 57 & NI FTP & {$[50, \mathrm{CJB}]$} \\
\hline 49 & 61 & RAND Network Graphics Conference & {$[30, \mathrm{MO} 2]$} \\
\hline 51 & 63 & Message Generator Control & {$[52, \mathrm{DFP}]$} \\
\hline 53 & 65 & AUTODIN II FTP & {$[21, \mathrm{EC} 5]$} \\
\hline 55 & 67 & ISI Graphics Language & {$[3, \mathrm{RB} 6]$} \\
\hline 57 & 71 & MTP & {$[45, \mathrm{JBP}]$} \\
\hline 59 & 73 & New MIT Host status & {$[\mathrm{SWG}]$} \\
\hline $61-63$ & $75-77$ & Unassigned & [ JBP ] \\
\hline
\end{tabular}


Port or Socket Numbers

Host Specific Functions

\begin{tabular}{|c|c|c|}
\hline Decimal & Octal & Description \\
\hline------- & ----- & ------------ \\
\hline 65 & 101 & Unassigned \\
\hline 67 & 103 & Datacomputer at $\mathrm{CCA}$ \\
\hline 69 & 105 & Unassigned \\
\hline 69 & 105 & Trivial File Transfer \\
\hline 71 & 107 & NETRJS (EBCDIC) at UCLA-CCN \\
\hline 73 & 111 & NETRJS (ASCII-68) at UCLA-CCN \\
\hline 75 & 113 & NETRJS (ASCII-63) at UCLA-CCN \\
\hline 77 & 115 & any private RJE server \\
\hline 79 & 117 & Name or Finger \\
\hline 81 & 121 & Unassigned \\
\hline 83 & 123 & MIT ML Device \\
\hline 85 & 125 & MIT ML Device \\
\hline 87 & 127 & any terminal link \\
\hline 89 & 131 & SU/MIT Telnet Gateway \\
\hline 91 & 133 & MIT Dover Spooler \\
\hline 93 & 135 & BBN RCC Accounting \\
\hline 95 & 137 & SUPDUP \\
\hline 97 & 141 & Datacomputer status \\
\hline 99 & 143 & CADC - NIFTP via UCL \\
\hline 101 & 145 & NPL - NIFTP via UCL \\
\hline 103 & 147 & BNPL - NIFTP via UCL \\
\hline 105 & 151 & CAMBRIDGE - NIFTP via UCL \\
\hline 107 & 153 & HARWELL - NIFTP via UCL \\
\hline 109 & 155 & SWURCC - NIFTP via UCL \\
\hline 111 & 157 & ESSEX - NIFTP via UCL \\
\hline 113 & 161 & RUTHERFORD - NIFTP via UCL \\
\hline $115-129$ & $163-201$ & Unassigned \\
\hline 131 & 203 & Datacomputer \\
\hline Reserved & for Future & Use \\
\hline Decimal & Octal & Description \\
\hline------- & ----- & ----------- \\
\hline $132-223$ & $204-337$ & Reserved \\
\hline
\end{tabular}

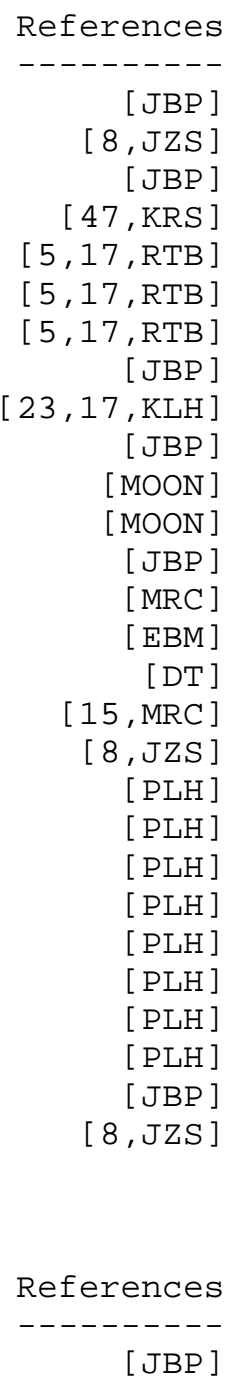


Port or Socket Numbers

Experimental Functions

$\begin{array}{lllr}\text { Decimal } & \text { Octal } & \text { Description } & \text { References } \\ ------ & ---- & -------- & ------- \\ 224-239 & 340-357 & \text { Unassigned } & \text { [ JBP ] } \\ 241 & 361 & \text { NCP Measurement } & \text { [9, JBP ] } \\ 243 & 363 & \text { Survey Measurement } & \text { [2, AV] } \\ 245 & 365 & \text { LINK } & \text { [7, RDB2 ] } \\ 247 & 367 & \text { TIPSRV } & \text { [RHT] } \\ 249-255 & 371-377 & \text { RSEXEC } & \text { [51, RHT] }\end{array}$

ASSIGNED LINK NUMBERS

The word "link" here refers to a field in the original ARPANET

Host/IMP interface leader. The link was originally defined as an 8 bit field. Some time after the ARPANET Host-to-Host (AHHP) protocol was defined and, by now, some time ago the definition of this field was changed to "Message-ID" and the length to 12 bits. The name link now refers to the high order 8 bits of this 12 bit message-id field. The low order 4 bits of the message-id field are to be zero unless specifically specified otherwise for the particular protocol used on that link. The Host/IMP interface is defined in BBN report 1822 [1].

Link Assignments:

\begin{tabular}{|c|c|c|c|}
\hline Decimal & $\begin{array}{l}\text { Octal } \\
-----\end{array}$ & Description & References \\
\hline 0 & 0 & AHHP Control Messages & {$[28,17, \mathrm{JBP}]$} \\
\hline 1 & 1 & Reserved & {$[\mathrm{JBP}]$} \\
\hline $2-71$ & $2-107$ & AHHP Regular Messages & {$[28,17, \mathrm{JBP}]$} \\
\hline $72-150$ & $110-226$ & Reserved & {$[\mathrm{JBP}]$} \\
\hline 151 & 227 & CHAOS Protocol & {$[\mathrm{MOON}]$} \\
\hline 152 & 230 & PARC Universal Protocol & {$[4, \operatorname{EAT} 3]$} \\
\hline 153 & 231 & TIP status Reporting & {$[\mathrm{JGH}]$} \\
\hline 154 & 232 & TIP Accounting & {$[\mathrm{JGH}]$} \\
\hline 155 & 233 & Internet Protocol (regular) & {$[33, \mathrm{JBP}]$} \\
\hline $156-158$ & $234-236$ & Internet Protocol (experimental) & {$[33, \mathrm{JBP}]$} \\
\hline $159-191$ & $237-277$ & Measurements & {$[9, \mathrm{VGC}]$} \\
\hline $192-195$ & $300-303$ & Unassigned & [ JBP ] \\
\hline $196-255$ & $304-377$ & Experimental Protocols & [ JBP ] \\
\hline $224-255$ & $340-377$ & NVP & {$[12,17, \mathrm{DC}]$} \\
\hline $248-255$ & $370-377$ & Network Maintenance & [ JGH ] \\
\hline
\end{tabular}




\section{DOCUMENTS}

[1] BBN, "Specifications for the Interconnection of a Host and an IMP", Report 1822, Bolt Beranek and Newman, Cambridge, Massachusetts, May 1978 .

[2] Bhushan, A., "A Report on the Survey Project", RFC 530, NIC 17375, 22 June 1973.

[3] Bisbey, R., D. Hollingworth, and B. Britt, "Graphics Language (version 2.1)", ISI/TM-80-18, USC/Information Sciences Institute, July 1980 .

[4] Boggs, D., J. Shoch, E. Taft, and R. Metcalfe, "PUP: An Internetwork Architecture", XEROX Palo Alto Research Center, CSL-79-10, July 1979; also in IEEE Transactions on Communication, Volume COM-28, Number 4, April 1980.

[5] Braden, R., "NETRJS Protocol", RFC 740, NIC 42423, 22 November 1977. Also in [17].

[6] Bressler, B., "Remote Job Entry Protocol", RFC 407, NIC 12112, 16 October 72. Also in [17].

[7] Bressler, R., "Inter-Entity Communication -- An Experiment", RFC 441, NIC 13773, 19 January 1973.

[8] CCA, "Datacomputer Version 5/4 User Manual", Computer Corporation of America, August 1979.

[9] Cerf, V., "NCP Statistics", RFC 388, NIC 11360, 23 August 1972 .

[10] Clark, D., "Revision of DSP Specification", Local Network Note 9, Laboratory for Computer Science, MIT, 17 June 1977.

[11] Clements, R., "FTPSRV -- Extensions for Tenex Paged Files", RFC 683, NIC 32251, 3 April 1975. Also in [17].

[12] Cohen, D., "Specifications for the Network Voice Protocol (NVP)", NSC Note 68, 29 January 1976. Also as USC/Information Sciences Institute RR-75-39, March 1976, and as RFC 741, NIC 42444, 22 November 1977. Also in [17].

[13] Cohen, D. and J. Postel, "Multiplexing Protocol", IEN 90, USC/Information Sciences Institute, May 1979. 
COMPASS, "Semi-Annual Technical Report", CADD-7603-0411, Massachusetts Computer Associates, 4 March 1976. Also as, "National Software Works, Status Report No. 1", RADC-TR-76-276, Volume 1, September 1976. And COMPASS. "Second Semi-Annual Report", CADD-7608-1611, Massachusetts Computer Associates, 16 August 1976.

[15] Crispin, M., "SUPDUP Protocol", RFC 734, NIC 41953, 7 October 1977. Also in [17].

[16] Crispin, M. and I. Zabala, "DIALNET Protocols", Stanford University Artificial Intelligence Laboratory, July 1978.

[17] Feinler, E. and J. Postel, eds., "ARPANET Protocol Handbook", NIC 7104, for the Defense Communications Agency by SRI International, Menlo Park, California, Revised January 1978.

[18] Flood Page, D., "Gateway Monitoring Protocol", IEN 131, February 1980 .

[19] Flood Page, D., "CMCC Performance Measurement Message Formats", IEN 157, September 1980.

[20] Forgie, J., "ST - A Proposed Internet Stream Protocol", IEN 119, M.I.T. Lincoln Laboratory, September 1979.

[21] Forsdick, H., and A. McKenzie, "FTP Functional specification", Bolt Beranek and Newman, Report 4051, August 1979.

[22] Harrenstien, K., J. Postel, "Time Server", IEN 142, April 1980. Also in [17].

[23] Harrenstien, K., "Name/Finger", RFC 742, NIC 42758, 30 December 1977. Also in [17].

[24] Harvey, B., "One More Try on the FTP", RFC 691, NIC 32700, 6 June 1975 .

[25] Haverty, J., "XNET Formats for Internet Protocol Version 4", IEN 158, October 1980 .

[26] McCarthy, J. and L. Earnest, "DIALNET", Stanford University Artificial Intelligence Laboratory, Undated.

[27] McKenzie, A., "File Transfer Protocol", RFC 454, NIC 14333, 16 February 1973. 
[28] McKenzie,A., "Host/Host Protocol for the ARPA Network", NIC 8246, January 1972. Also in [17].

[29] NSW Protocol Committee, "MSG: The Interprocess Communication Facility for the National Software Works", CADD-7612-2411, Massachusetts Computer Associates, BBN 3237, Bolt Beranek and Newman, Revised 24 December 1976.

[30] O'Brien, M., "A Network Graphical Conferencing System", RAND Corporation, N-1250-ARPA, August 1979.

[31] Postel, J., "Character Generator Process", RFC 429, NIC 13281, 12 December 1972 .

[32] Postel, J., "Discard Process", RFC 348, NIC 10427, 30 May 1972 .

[33] Postel, J., ed., "Internet Protocol - DARPA Internet Program Protocol Specification", RFC 791, USC/Information Sciences Institute, September 1981.

[34] Postel, J., ed., "Transmission Control Protocol - DARPA Internet Program Protocol Specification", RFC 793, USC/Information Sciences Institute, September 1981.

[35] Postel, J., "Echo Process", RFC 347, NIC 10426, 30 May 1972.

[36] Postel, J., "File Transfer Protocol", RFC 765, IEN 149, June 1980 .

[37] Postel, J., "Internet Message Protocol", RFC 759, IEN 113, USC/Information Sciences Institute, August 1980.

[38] Postel, J., "Name Server", IEN 116, USC/Information Sciences Institute, August 1979.

[39] Postel, J., "Official Initial Connection Protocol", NIC 7101, 11 June 1971. Also in [17].

[40] Postel, J., "Telnet Protocol", RFC 318, NIC 9348, 3 April 1972 .

[41] Postel, J., "Telnet Protocol Specification", RFC 764, IEN 148, June 1980 .

[42] Postel, J., "User Datagram Protocol", RFC 768 USC/Information Sciences Institute, August 1980. 
[43] Reed, D., "Protocols for the LCS Network", Local Network Note 3, Laboratory for Computer Science, MIT, 29 November 1976.

[44] Skelton, A., S. Holmgren, and D. Wood, "The MITRE Cablenet Project", IEN 96, April 1979.

[45] Sluizer, S., and J. Postel, "Mail Transfer Protocol", RFC 780, USC/Information Sciences Institute, May 1981.

[46] Sproull, R., and E. Thomas. "A Networks Graphics Protocol", NIC 24308, 16 August 1974. Also in [17].

[47] Sollins, K., "The TFTP Protocol (revision 2)", RFC 783, MIT/LCS, June 1981.

[48] Strazisar, V., "Gateway Routing: An Implementation Specification", IEN 30, Bolt Berenak and Newman, April 1979.

[49] Strazisar, V., "How to Build a Gateway", IEN 109, Bolt Berenak and Newman, August 1979.

[50] The High Level Protocol Group, "A Network Independent File Transfer Protocol", INWG Protocol Note 86, December 1977.

[51] Thomas, R., "A Resource Sharing Executive for the ARPANET", AFIPS Conference Proceedings, 42:155-163, NCC, 1973.

[52] Flood Page, D., "A Simple Message Generator", IEN 172, Bolt Berenak and Newman, March 1981.

[53] Postel, J., "Internet Control Message Protocol - DARPA Internet Program Protocol Specification", RFC 792, USC/Information Sciences Institute, September 1981.

[54] Postel, J., "Simple Mail Transfer Protocol", RFC 788, USC/Information Sciences Institute, september 1981.

[55] Littauer, B., "A Host Monitoring Protocol"", IEN 197, Bolt Berenak and Newman, September 1981. 


\begin{tabular}{|c|c|c|c|}
\hline & & $\begin{array}{l}\text { PEOPLE } \\
------\end{array}$ & \\
\hline [DCA2 ] & Don Allen & $\mathrm{BBN}$ & Allen@BBND \\
\hline [CJB] & Chris Bennett & $\mathrm{UCL}$ & UKSAT@ISIE \\
\hline$[\mathrm{RB} 6]$ & Richard Bisbey & ISI & Bisbey@ISIB \\
\hline [RTB] & Bob Braden & UCLA & Braden@ISIA \\
\hline [RDB2 ] & Robert Bressler & $\mathrm{BBN}$ & BresslereBBNE \\
\hline [EC5 ] & Ed Cain & $\mathrm{DCEC}$ & cain@EDN-Unix \\
\hline [VGC] & Vint Cerf & ARPA & CerfeISIA \\
\hline [NC3] & J. Noel Chiappa & MIT & JNC@MIT-XX \\
\hline$[\mathrm{SGC}]$ & Steve Chipman & $\mathrm{BBN}$ & Chipman@BBNA \\
\hline [DDC2 ] & David Clark & MIT & Clark@MIT-Multics \\
\hline$[\mathrm{DC}]$ & Danny Cohen & ISI & CoheneISIB \\
\hline [MRC ] & Mark Crispin & Stanford & Admin.MRC@SU-SCORE \\
\hline [BD2 ] & Brian Davies & RSRE & T45@ISIE \\
\hline [ JAKE ] & Jake Feinler & SRI & Feinler@SRI-KL \\
\hline$[\mathrm{DFP}]$ & David Flood Page & $\mathrm{BBN}$ & DFloodPage@BBNE \\
\hline [ JWF ] & Jim Forgie & LL & Forgie@BBNC \\
\hline [SWG ] & Stu Galley & MIT & SWG@MIT-DMS \\
\hline [GEOF ] & Geoff Goodfellow & SRI & GeoffeDARCOM-KA \\
\hline$[\mathrm{KLH}]$ & Ken Harrenstien & MIT & KLH@MIT-AI \\
\hline [ JFH2 ] & Jack Haverty & $\mathrm{BBN}$ & JHaverty@BBN-Unix \\
\hline [ JGH ] & Jim Herman & $\mathrm{BBN}$ & Herman@BBNE \\
\hline$[\mathrm{PLH}]$ & Peter Higginson & UCL & UKSAT@ISIE \\
\hline$[\mathrm{RH} 6]$ & Robert Hinden & $\mathrm{BBN}$ & Hinden@BBNE \\
\hline$[\mathrm{CH} 2]$ & Charles Hornig & Honeywell & Hornig@MIT-Multics \\
\hline$[\mathrm{EAK}]$ & Earl Killian & LLL & EAK@MIT-MC \\
\hline$[\mathrm{PK}]$ & Peter Kirstein & UCL & Kirstein@ISIA \\
\hline [DRL ] & David Lyons & $\mathrm{DEC}$ & Lyons@DEC-2136 \\
\hline [ HM] & Hank Magnuski & --- & --- \\
\hline [ JEM] & Jim Mathis & SRI & Mathis@SRI-KL \\
\hline [DM11 ] & Dale McNeill & $\mathrm{BBN}$ & DMcNeill@BBNE \\
\hline [DLM1 ] & David Mills & COMSAT & Mills@ISIE \\
\hline$[\mathrm{MOON}]$ & David Moon & $\mathrm{MIT}$ & Moon@MIT-MC \\
\hline$[\mathrm{EBM}]$ & Eliot Moss & MIT & EBM@MIT-XX \\
\hline [MO2] & Michael O'Brien & RAND & OBrien@RAND-Unix \\
\hline$[\mathrm{KTP}]$ & Ken Pogran & $\mathrm{BBN}$ & Pogran@BBND \\
\hline$[\mathrm{JBP}]$ & Jon Postel & ISI & Postel@ISIF \\
\hline$[\mathrm{JZS}]$ & Joanne Sattely & $\mathrm{CCA}$ & JZS@CCA \\
\hline$[\mathrm{APS}]$ & Anita Skelton & MITRE & skelton@MITRE \\
\hline [KRS ] & Karen Sollins & $\mathrm{MIT}$ & Sollins@MIT-XX \\
\hline [VMS ] & Virginia Strazisar & $\mathrm{BBN}$ & Strazisar@BBNA \\
\hline [EAT3] & Ed Taft & XEROX & Taft.PA@PARC \\
\hline [DT ] & Dan Tappan & $\mathrm{BBN}$ & Tappan@BBNG \\
\hline$[\mathrm{RHT}]$ & Robert Thomas & $\mathrm{BBN}$ & Thomas@BBNA \\
\hline$[\mathrm{AV}]$ & Al Vezza & $\mathrm{MIT}$ & AV@MIT-XX \\
\hline [ CJW2 ] & Cliff Weinstein & LL & c jw@LL-11 \\
\hline
\end{tabular}

